\title{
MATRICES WHOSE CHARACTERISTIC EQUATIONS ARE CYCLIC*
}

\author{
BY T. A. PIERCE
}

One of Sylvester's theorems $†$ on matrices states that if the characteristic equation

$$
|M-\lambda I|=f(\lambda)=0
$$

of a square matrix $M$ has the roots $\lambda_{1}, \lambda_{2}, \cdots, \lambda_{n}$, then the characteristic equation

$$
|\phi(M)-\rho I|=g(\rho)=0
$$

of any integral function of $M$, namely, $\phi(M)$, has the roots $\rho_{i}=\phi\left(\lambda_{i}\right), i=1,2, \cdots, n$. In this note an isomorphism is shown to exist between the algebraic and matric roots of (1) when this equation is cyclic. Certain consequences of this isomorphism are given. Since

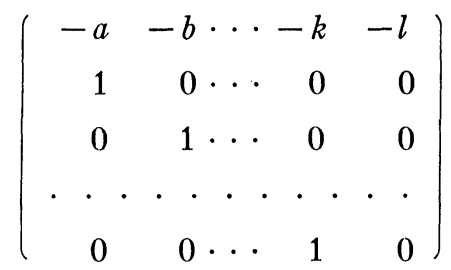

is the matrix which has $\lambda^{n}+a \lambda^{n-1}+b \lambda^{n-2}+\cdots+k \lambda+l=0$ as its characteristic equation, $\ddagger$ Sylvester's theorem furnishes a method of effecting the Tschirnhaus transformation $\rho=\phi(\lambda)$ on any equation. When (1) is cyclic an especially interesting type of Tschirnhaus transformation is possible.

Suppose (1) to be a cyclic equation of degree $n$ with the relations $\lambda_{i+1}=\phi\left(\lambda_{i}\right), i=1,2, \cdots, n$ and $\lambda_{n+j}=\lambda_{j}$ connecting its roots. For simplicity of notation let $\phi_{i}$ be the $i$ th iterated function of $\phi$ so that $\lambda_{2}=\phi\left(\lambda_{1}\right)=\phi_{1}\left(\lambda_{1}\right), \lambda_{3}=\phi\left(\lambda_{2}\right)=\phi\left(\phi\left(\lambda_{1}\right)\right)$ $=\phi_{2}\left(\lambda_{1}\right)$, and in general $\lambda_{i+1}=\phi_{i}\left(\lambda_{1}\right)$. By Sylvester's theorem the roots of (2) then are

* Presented to the Society, December 30, 1929.

† Sylvester, Mathematical Papers, vol. 4, p. 133; Frobenius, Journal für Mathematik, vol. 84, p. 11; Dickson, Algebren und ihre Zahlentheorie, p. 18.

‡Wedderburn, Annals of Mathematics, vol. 27 (1926), p. 247. 
$\rho_{1}=\phi\left(\lambda_{1}\right)=\lambda_{2}, \rho_{2}=\phi\left(\lambda_{2}\right)=\lambda_{3}, \cdots, \rho_{n}=\phi\left(\lambda_{n}\right)=\lambda_{n+1}=\lambda_{1}$.

Thus the roots of (1) and (2) are the same, and $M$ and $\phi(M)$ have the same characteristic equation. Similarly, if we form the characteristic equation of $\phi_{i}(M)$, we find that its roots are

$\rho_{1}=\phi_{i}\left(\lambda_{1}\right)=\lambda_{i+1}, \quad \rho_{2}=\phi_{i}\left(\lambda_{2}\right)=\lambda_{i+2}, \cdots, \rho_{n}=\phi_{i}\left(\lambda_{n}\right)=\lambda_{i}$, where the subscripts are to be reduced modulo $n$. Hence we have the following theorem.

THEOREM 1. If the characteristic equation, $f(\lambda)=0$, of the matrix $M$ is cyclic with the relations $\lambda_{i}=\phi_{i}\left(\lambda_{1}\right), i=1,2, \cdots, n$, connecting its roots, then the matrices $M, \phi_{1}(M), \cdots, \phi_{n-1}(M)$, all have the same characteristic equation.

Applying the Hamilton-Cayley theorem we have the following additional theorem.

THEOREM 2. Under the conditions of Theorem 1 above $f(\lambda)=0$ has its full complement of matric roots, namely,

$$
M, \phi_{1}(M), \cdots, \phi_{n-1}(M) .
$$

The matric roots of this last theorem may be called conjugate* and may be denoted by

$$
M_{1}, M_{2}=\phi_{1}\left(M_{1}\right), \cdots, M_{n}=\phi_{n-1}\left(M_{1}\right) .
$$

Since these matrices are all functions of one matrix they are commutative under multiplication and division, and since the same cyclic relations exist among the $M$ 's as among the $\lambda$ 's, we have the following theorem.

THEOREM 3. The field generated by the roots of any cyclic equation is isomorphic to the field generated by the matric roots of this same equation.

Consider now any integral function of the matrices $M_{1}, \cdots, M_{n}$, namely $F\left(M_{1}, \cdots, M_{n}\right)$, and form its characteristic equation

$$
\left|F\left(M_{1}, \cdots, M_{n}\right)-\rho I\right|=0 .
$$

Now $F\left(M_{1}, \cdots, M_{n}\right)=G\left(M_{1}\right)$ by virtue of (3), and hence the roots of (4) are by Sylvester's theorem $\rho_{i}=G\left(\lambda_{i}\right), i=1,2, \cdots, n$. But

* Taber, American Journal of Mathematics, vol. 13, p. 159. 


$$
\begin{aligned}
\rho_{1}=G\left(\lambda_{1}\right) & =F\left[\lambda_{1}, \phi_{1}\left(\lambda_{1}\right), \cdots, \phi_{n-1}\left(\lambda_{1}\right)\right] \\
& =F\left[\lambda_{1}, \lambda_{2}, \cdots, \lambda_{n}\right], \\
\rho_{2}=G\left(\lambda_{2}\right) & =F\left[\lambda_{2}, \phi_{1}\left(\lambda_{2}\right), \cdots, \phi_{n-1}\left(\lambda_{2}\right)\right] \\
& =F\left[\lambda_{2}, \lambda_{3}, \cdots, \lambda_{1}\right],
\end{aligned}
$$

and in general we find that

$$
\rho_{i}=G\left(\lambda_{i}\right)=F\left[\lambda_{i}, \lambda_{i+1}, \cdots, \lambda_{i-1}\right] ;
$$

thus we obtain the theorem which follows:

TheOREM 4. Let the characteristic equation of $M_{1}$ be cyclic and have the roots $\lambda_{1}, \lambda_{2}, \cdots, \lambda_{n}$ and the corresponding matric roots $M_{1}, M_{2}, \cdots, M_{n}$. Then, if $F\left(M_{1}, \cdots, M_{n}\right)$ be an integral function, the roots of the characteristic equation of $F\left(M_{1}, \cdots, M_{n}\right)$ are $F\left(\lambda_{1}, \cdots, \lambda_{n}\right)$ and the quantities obtained from this by permuting $\lambda_{1}, \lambda_{2}, \cdots, \lambda_{n}$ cyclically. The matric roots of this equation are obtained from $F\left(M_{1}, \cdots, M_{n}\right)$ by permuting $M_{1}, M_{2}, \cdots$, $M_{n}$ cyclically. Moreover this equation is cyclic.

Thus, for example, if $\left|M_{1}-\lambda I\right|=0$ is cyclic and has the roots $\lambda_{1}, \lambda_{2}, \cdots, \lambda_{n}$, the roots of $\left|M_{1}+M_{2}-\rho I\right|=0$ are $\rho_{1}=\lambda_{1}+\lambda_{2}$, $\rho_{2}=\lambda_{2}+\lambda_{3}, \cdots, \rho_{n}=\lambda_{n}+\lambda_{1}$. To the first equation the Tschirnhaus transformation $\rho=\lambda+\phi(\lambda)$ has been applied and this transformation affects the roots of the equation in pairs.

If $C_{m}$ denotes the $m$ th compound of the matrix $M$ it is known* that the roots of the characteristic equation

$$
\left|C_{m}-\rho I\right|=0
$$

are the products $m$ at a time of the roots of $|M-\lambda I|=0$. If the latter is a cyclic equation its roots are all functions of one root and the roots of (5) are therefore all functions of this root. By the isomorphism of Theorem 3 the roots of (5) correspond to matrices all of which are functions of one matrix and hence are commutative under multiplication. Moreover by Theorem 3 these matrices all satisfy (5) so that (5) has its full complement of matric roots. These matric roots are, however, in general of order different from the degree of (5).

The University of Nebraska

* Pascal, Repertorium der höheren Mathematik, vol. 2, 1910, p. 139; Whittaker, Proceedings of the Edinburgh Mathematical Society, vol. 35 (1916), p. 2. 